\title{
The Roles of c-Jun N-terminal Kinase (JNK) and p38 Mitogen-activated Protein Kinase (p38 MAPK) in Aged Pig Oocytes
}

\author{
Ivana PETROVÁ ${ }^{1)}$, Markéta SEDMÍKOVÁ(2), Jaroslav PETR ${ }^{3)}$, Zuzana VODKOVÁ(2), \\ Petr PYTLOUN ${ }^{2)}$, Eva CHMELÍKOVÁ2), Dalibor ŘEHÁK ${ }^{3)}$, Andrea ĆTRNÁCTÁ ${ }^{2)}$, \\ Radko RAJMON ${ }^{2)}$ and František JíLEK ${ }^{2)}$
}

\begin{abstract}
1) Faculty of Science, Charles University, 12843 Prague 2, 2) Department of Veterinary Sciences, Czech University of Life Sciences in Prague, 16521 Prague 6 - Suchdol and ${ }^{3)}$ Research Institute of Animal Production, 10401 Prague 10-Uhríne ves, Czech Republic
\end{abstract}

\begin{abstract}
After reaching metaphase II, in vitro matured oocytes undergo the complex processes referred to as oocyte aging. Under our culture conditions, some aged oocytes remained at the stage of metaphase II, some underwent spontaneous parthenogenetic activation and others underwent cellular death, either through apoptosis (fragmentation) or lysis. We investigated the effect of c-Jun N-terminal kinases (JNK) and p38 Mitogen-activated protein kinase (p38 MAPK) inhibition on pig oocyte aging and the activity of JNK and p38 MAPK during the aging period. Inhibition of JNK protected the oocytes from fragmentation $(0 \%$ fragmented oocytes under JNK inhibition vs. $26 \%$ fragmented oocytes in the control group). Inhibition of p38 MAPK had no effect on fragmentation. Inhibition of JNK also had an influence on spontaneous parthenogenetic activation of aged oocytes. The ratio of activated JNK to total JNK decreased during aging of oocytes. However, exit from MII had no effect on it. The ratio of activated p38 MAPK to total p38 MAPK did not change significantly. The phosphorylated form of JNK is present in fragmented and activated oocytes, while lysed oocytes lack the active form of JNK. Based on our data, we can conclude that JNK plays an active role in fragmentation of pig oocytes and that p38 MAPK is not involved in this process.
\end{abstract}

Key words: Aging, c-Jun N-terminal kinases (JNK), p38 Mitogen-activated protein kinase (p38 MAPK), Oocyte, Pig

(J. Reprod. Dev. 55: 75-82, 2009)

$\boldsymbol{A}$ rich source of high-quality oocytes is essential for reproductive biotechnologies. In vitro matured oocytes are frequently used for in vitro fertilization, cloning and transgenesis. The meiotic maturation of oocytes is completed at the stage of metaphase II, when oocyte meiosis is spontaneously blocked [1]. Exit from this meiotic block depends on an activating stimulus, which induces destruction or deactivation of the molecules responsible for maintenance of meiotic block [2].

The quality of in vitro matured oocytes often does not meet all the requirements for successful performance of various biotechniques. This problem is sometimes dealt with by using prolonged in vitro culture of the oocytes. Oocytes cultured in vitro after reaching the stage of metaphase II have been used for in vitro fertilization [3, 4] cloning [5-7] and production of parthenogenetic embryos [8].

Prolonged in vitro culture of mammalian oocytes is accompanied by changes in stability of the oocyte cytoskeleton [9] and in the activity of various enzymes [10]. This complex process is referred to as aging. Aging can result in spontaneous parthenogenetic activation, fragmentation or lysis in pig oocytes [11-13]. All of these changes result in deterioration of the quality or viability of the oocytes and therefore interfere with their success in reproductive biotechnologies [14]. The nature of these processes is not completely understood. Programmed cell death, or apoptosis, is

Accepted for publication: October 15, 2008

Published online in J-STAGE: November 20, 2008

Correspondence: M Sedmíková (e-mail: sedmikova@af.czu.cz) involved in these processes [15-18], and it is manifested in the oocyte as fragmentation [16, 19, 20]. Apoptosis is triggered and accomplished by a wide spectrum of specific bioactive molecules, including caspases, proteases responsible for dismantling of the cell and many pro- and anti-apoptotic factors from the Bcl-2 gene family [21].

Sadler et al. [22] clearly demonstrated that induction of pro-apoptotic factors in starfish oocytes is inhibited by oocyte activation, which results in decreased activity of the maturation-promoting factor (MPF) and mitogen-activated protein kinases (MAPK). Without fertilization or parthenogenetic activation, elevated levels of MAPK induce apoptosis in the oocyte.

The family of MAPKs is involved in regulation of many signalling cascades in eukaryotic cells. Five groups of MAPK have so far been identified. The first group consists of extracellular signal-regulated kinase (ERKs) 1 and 2. The members of the second group are c-Jun amino terminal kinase (JNKs) 1,2 and 3. Isoforms $\alpha, \beta$, $\gamma$ and $\delta$ of kinase p38 (protein with an apparent molecular weight of $38 \mathrm{kDa}$ ) comprise the third group. ERK3 and ERK4 are in the fourth group. The fifth group of MAPKs consists of large MAP kinase 1 (BMK1 or ERK5) [23, 24]. ERKs play an important role in cell proliferation and differentiation. JNK and p38 MAPK are involved in the stress response of the cell [25]. JNK and p38 MAPK have been found in Xenopus and mouse oocytes [26, 27], and p38 MAPK has also been found in porcine oocytes, where it is involved in meiotic maturation [28].

In aged starfish oocytes, the stress kinases JNK and p38 MAPK 

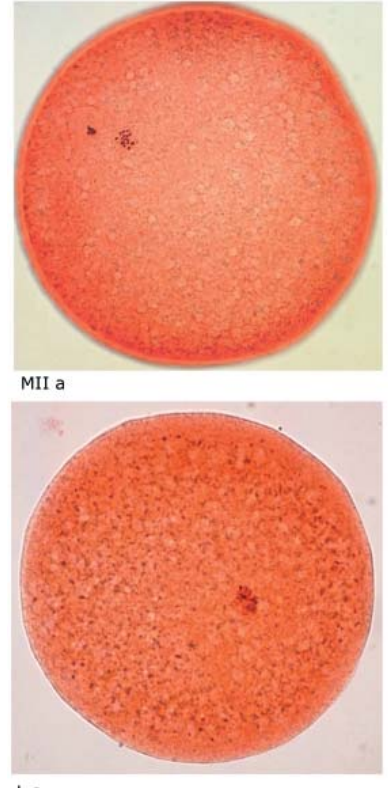

L a

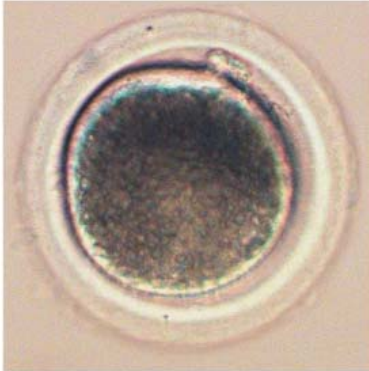

MII b

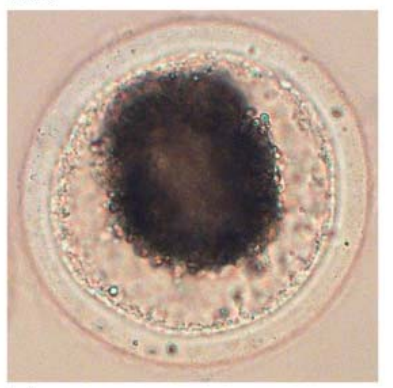

L b

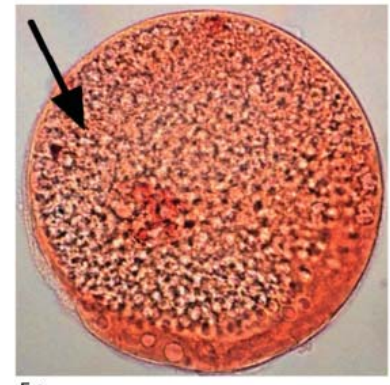

$\mathrm{Fa}$

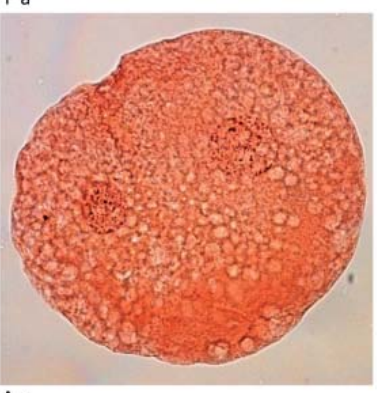

A a

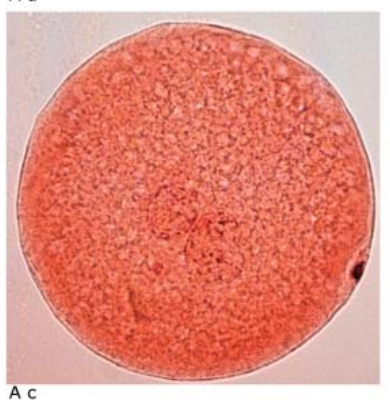

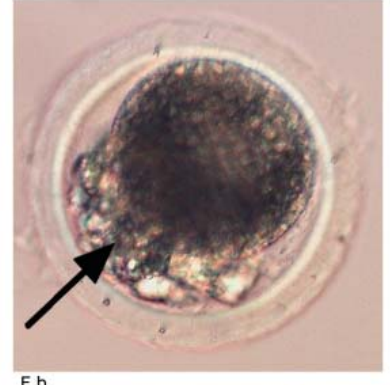

$\mathrm{Fb}$

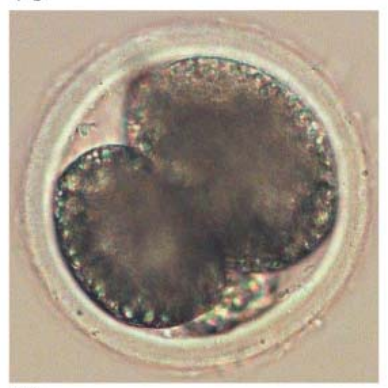

A b

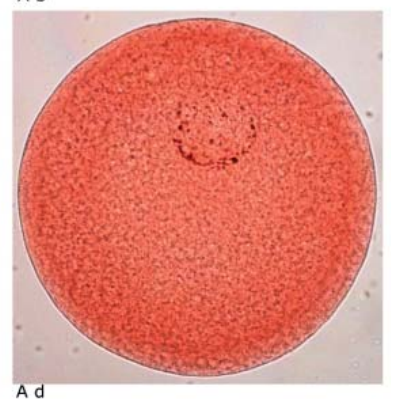

Fig. 1. Categories of aged pig oocytes. MIIa, MIIb-metaphase II oocyte (1 day of aging), Fa, Fb-fragmented oocyte (3 days of aging, fragment vesicles are indicated by arrow), La, Lb-lysed oocyte (3 days of aging), Ac, Adactivated oocytes (oocytes with pronuclei, 3 days of aging) and $\mathrm{Aa}, \mathrm{Ab}$-activated oocytes (cleaved oocytes, 3 days of aging). MIIa, Fa, La, Ab, Ac and Ad-oocytes were fixed and stained with orcein (magnification 40×). MIIb, Lb, Fb and Ab oocytes were scanned immediately after cultivation without staining (magnification 20×).

play a key role in oocyte fragmentation [22]. These stress MAPKs are also involved in regulation of many intracellular activities [24], including programmed cell death through apoptosis [29-31].

The roles of JNK and p38 MAPK in oocyte aging in vertebrate oocytes are not fully understood. Sadler et al. [22] assumed that a similar role of these kinases in starfish and mammals would open up the possibility of therapeutic prolongation of mammalian oocyte viability through inhibition of these kinases. However, the validity of this hypothesis remains to be verified.

The objective of this study was to investigate the activity of JNK and p38 MAPK during oocyte aging and to test the effects of JNK and p38 MAPK inhibition on the processes involved in aging of pig oocytes.

\section{Materials and Methods}

\section{Isolation and culture of oocytes}

Pig ovaries were obtained from a local slaughterhouse from gilts at an unknown stage of the estrous cycle and transported to the laboratory within $1 \mathrm{~h}$ in a saline solution ( $0.9 \%$ sodium chloride) at 39 C. Fully-grown oocytes were collected by aspirating follicles that were $2-5 \mathrm{~mm}$ in diameter with a 20-gauge needle. Only oocytes with compact cumuli were selected for further study. Before culture, the oocytes were washed three times in a maturation culture

medium.

The oocytes were cultured in a modified M199 medium (GibcoBRL, Life Technologies, Paisley, Scotland) containing sodium bicarbonate $(32.5 \mathrm{mM})$, calcium L-lactate $(2.75 \mathrm{mM})$, gentamicin (0.025 mg/ml), HEPES (6.3 mM), 13.5 IU eCG: 6.6 IU hCG/ml (P.G.600; Intervet, Boxmeer, Holland) and 10\% (v/v) fetal calf serum (GibcoBRL). The oocytes were cultured for $48 \mathrm{~h}$ in $3.5-\mathrm{cm}$ diameter Petri dishes (Nunc, Roskilde, Denmark) containing $3.0 \mathrm{ml}$ of the culture medium at $39 \mathrm{C}$ in a mixture of $5.0 \% \mathrm{CO}_{2}$ in air.

\section{Evaluation of oocytes}

At the end of culture, the oocytes or embryos were mounted on slides, fixed with acetic alcohol (1:3, v/v) for at least $24 \mathrm{~h}$ and stained with $1.0 \%(\mathrm{w} / \mathrm{v})$ orcein. They were then examined under a microscope and classified into four groups [13]. Activation was considered to have occurred if the oocytes had reached the pronuclear stage. Oocytes remaining at metaphase II or arrested at anaphase II or telophase II were not considered to be activated. In cleaved parthenogenetic embryos, the numbers of nuclei were counted at the end of culture. Oocytes were designated as fragmented when fragmented "vesicles" were observed under the zona pellucida. "Fragmented vesicles" are indicated by arrows in Fig. 1 and are also described in the figure legend. Rupture of the cytoplasmic membrane and loss of integrity of the oocyte were the main 
criteria for lysis (Fig. 1).

\section{Determination of JNK and $p 38$ MAPK activity}

Phosphorylation of tyrosine and threonine is necessary for activation of JNK and p38 MAPK [20]. The Cellular Activation of Signalling ELISA (CASE) kit (SuperArray, Frederick, MD, USA) was used to determine JNK and p38 MAPK activity. The oocytes were cultured in a modified M199 medium (as described above) and were denuded of cumulus cells. The zona pellucida was removed by $0.1 \mathrm{~N} \mathrm{HCl}$, and the oocytes were fixed with a fixing buffer [9.4 ml of PBS, $2.6 \mathrm{ml}$ of 3.7\% (w/v) formaldehyde and 10 $\mathrm{ml}$ of the cultivation medium]. The fixed oocytes were stored at 4 $\mathrm{C}$ until processing. Determination of JNK and p38 MAPK activity was performed according to the SuperArray CASE kit user's manual as follows. The fixed oocytes (50 and 20 oocytes in each sample for determination of JNK and p38 MAPK, respectively) were incubated with primary antibodies. Two primary antibodies were used, one for the phosphorylated form of proteins (anti-phospho-JNK antibody, dilution 1:150, and anti-phospho-p38 MAPK antibody, dilution 1:100) and the other for the total target proteins (anti-pan-JNK antibody, dilution 1:200, and anti-pan-p38 MAPK antibody, dilution 1:100). The oocytes were then incubated with secondary antibodies (dilution 1:160 and 1:16 for determination of JNK and p38 MAPK, respectively), and the absorbance was measured (wavelength $450 \mathrm{~nm}$ ) by an ELISA plate reader (Rainbox Reader, SLT Laboratory Instruments, Salzburg, Austria). The specificity of the antibodies for JNK and p38 MAPK activity in pig oocytes was confirmed by Western blot (data not shown).

\section{Laser-scanning confocal microscopy}

After the aging period, the zonae pellucidae of the oocytes and embryos were removed by treatment in $0.1 \%(\mathrm{w} / \mathrm{v})$ pronase (Sigma-Aldrich, St. Louis, MO, USA). The oocytes and embryos were then washed three times in PBS-BSA and fixed in $2.5 \%(\mathrm{w} / \mathrm{v})$ paraformaldehyde in PBS for 60 min. After treatment in PBSBSA-Triton X-100 (Sigma-Aldrich) for $2 \mathrm{~h}$, they were incubated in PBS-BSA-Tween 20 (Sigma-Aldrich) containing mouse monoclonal anti-phospho-JNK (1:100, Sigma-Aldrich) at 4 C overnight. After being washed three times in PBS-Tween 20 (10 min each), the oocytes and embryos were incubated in PBS-BSA-Tween 20 containing fluorescein isothiocyanate (FITC)-conjugated goat antimouse IgG (1:100, Sigma-Aldrich) for $60 \mathrm{~min}$ at room temperature. Negative control experiments were performed by first antibody omission. After repeated washing in PBS-Tween 20 (three times, 10 min each), the DNA was stained with Hoechst 33258 (0.5\% w/ v, Sigma-Aldrich, $10 \mathrm{~min})$. The oocytes and embryos were mounted on slides using a SlowFade antifade kit (Invitrogen, Carlsbad, CA, USA) and observed under a laser-scanning confocal microscope (Leica TCS SPE, Leica Microsystems, Wetzlar, Germany).

\section{Experimental design}

The purpose of Experiment 1 was to investigate the fates of pig oocytes aged in vitro. In vitro matured oocytes were denuded of cumulus cells, placed in an M199 culture medium and cultured for another 1, 2, 3, 4 or 5 days. At the end of culture, the oocytes were evaluated as described above.

Experiment 2 was performed to investigate the effects of JNK inhibition on the aging of pig oocytes. Oocytes matured in vitro were cultured for another 3 days in a medium supplemented with the following concentrations of JNK 1,9-pyrazoloanthrone (Calbiochem): 0, 5, 10, 20 or $30 \mu \mathrm{M}$. Control experiments were performed on oocytes aged for 3 days in an inhibitor-free medium. In another control experiment, the in vitro matured oocytes were cultured with $\mathrm{N}^{1}$-methyl-1,9-pyrazoloanthrone (Calbiochem, concentrations of 0 , $5,10,20$ or $30 \mu \mathrm{M}$ ), which is known as an inactive form of JNK inhibitor 1,9-pyrazoloanthrone. At the end of culture, the oocytes were evaluated as described above.

Experiment 3 was performed to investigate the effects of p38 MAPK inhibition on the aging of pig oocytes. Oocytes matured in vitro were cultured for another 3 days in a medium supplemented with the following concentrations of the specific inhibitor of p38 MAPK 2-(4-chlorophenyl)-4-(4-fluorophenyl)-5-pyridin-4-yl-1,2 dihydropyrazol-3-one (Calbiochem): 0, 0.1, 0.5, 1, 2.5 and $5 \mu \mathrm{M}$. Control experiments were performed on oocytes aged for 3 days in an inhibitor-free medium. At the end of culture, the oocytes were evaluated as described above. The active and inactive forms of the JNK inhibitors and the p38 MAPK inhibitor were dissolved in DMSO and then added to the culture medium. The content of DMSO in the medium was no higher than $0.7 \%$. Control experiments were conducted using a medium with a corresponding amount of DMSO but without inhibitors.

In experiment 4 we assayed the activity of JNK and p38 MAPK in aging oocytes. Oocytes matured in vitro were cultured for another $0,1,2$ or 3 days, and the activity of the respective kinase was then assayed using a CASE kit as described above. The activity of kinases was evaluated by comparison of the absorbance of samples that were incubated with antibodies against a total target protein and samples that were incubated with antibodies against phosphorylated forms of proteins.

Experiment 5 was performed to investigate the JNK activity of activated MII oocytes. Three groups of oocytes matured in vitro were studied. MII oocytes comprised the first group. The second group of aged oocytes was cultured for another $12 \mathrm{~h}$. The third group was activated using calcium ionophore A23187 (Sigma-Aldrich, $25 \mu \mathrm{M}$ for $5 \mathrm{~min}$ ) and then cultured in a medium supplemented with $2 \mathrm{mM}$ of 6-dimethylaminopurine (Sigma-Aldrich) [32]. The activated oocytes were cultured for another $10 \mathrm{~h}$ to complete an overall culture time of $12 \mathrm{~h}$. The control group of 40 oocytes was checked $12 \mathrm{~h}$ after activation treatment, and the activation rate was $85 \%$. The activity of JNK in all three groups of oocytes was assayed using a CASE kit as described above.

Experiment 6 was performed to detect the subcellular localization of the phosphorylated form of JNK in aging oocytes. The oocytes matured in vitro were cultured for another 1, 2 or 3 days. There were at least twelve oocytes in each group. The localization of phosphorylated JNK in oocytes was assayed as described above.

\section{Statistical analysis}

Data from all the experiments were subjected to statistical analysis. Experiments 1, 2 and 3 were each performed four times. The differences between the groups of oocytes (days of aging, concen- 
Table 1. The fates of pig oocytes aged in vitro

\begin{tabular}{|c|c|c|c|c|}
\hline \multirow{2}{*}{$\begin{array}{l}\text { In vitro aging } \\
\text { (days) }\end{array}$} & \multicolumn{4}{|c|}{ Type of oocyte $(\% \pm \mathrm{SE})$} \\
\hline & Metaphase II & Activated & Fragmented & Lysed \\
\hline 1 & $98 \pm 1^{\mathrm{A}}$ & $2 \pm 1^{\mathrm{A}}$ & $0 \pm 0^{\mathrm{A}}$ & $0 \pm 0^{\mathrm{A}}$ \\
\hline 2 & $35 \pm 1^{\mathrm{B}}$ & $39 \pm 3^{\mathrm{BC}}$ & $17 \pm 1^{\mathrm{B}}$ & $9 \pm 1^{\mathrm{B}}$ \\
\hline 3 & $22 \pm 1^{\mathrm{C}}$ & $45 \pm 2^{\mathrm{C}}$ & $31 \pm 2^{\mathrm{C}}$ & $3 \pm 1^{\mathrm{A}}$ \\
\hline 4 & $16 \pm 1^{\mathrm{C}}$ & $30 \pm 2^{\mathrm{B}}$ & $24 \pm 2^{\mathrm{BC}}$ & $30 \pm 2^{C}$ \\
\hline 5 & $23 \pm 1^{\mathrm{C}}$ & $34 \pm 1^{\mathrm{B}}$ & $20 \pm 3^{\mathrm{B}}$ & $23 \pm 0^{\mathrm{D}}$ \\
\hline
\end{tabular}

Oocytes were matured in vitro for $48 \mathrm{~h}$ and then further cultured for another 1, 2, 3, 4 or 5 days. The total number of oocytes in each group is 120 . A, B, C Statistically significant differences between oocytes of different ages within the same type of oocyte (i.e., differences within columns) are indicated by different superscripts ( $<<0.05)$. The same letters indicate statistical insignificance.

Table 2. The effect of the JNK inhibitor 1,9-pyrazoloanthrone on the aging and spontaneous parthenogenetic activation of aged pig oocytes

\begin{tabular}{|c|c|c|c|c|c|c|}
\hline \multirow{2}{*}{$\begin{array}{l}\text { Concentration } \\
\text { of JNK inhibitor } \\
\qquad(\mu \mathrm{M})\end{array}$} & \multicolumn{6}{|c|}{ Type of oocyte $(\% \pm \mathrm{SE})$} \\
\hline & Metaphase II & Fragmented & Lysed & Activated (sum) & $\begin{array}{l}\text { Activated (oocytes } \\
\text { with pronuclei) }\end{array}$ & $\begin{array}{c}\text { Activated (cleaved } \\
\text { oocytes) }\end{array}$ \\
\hline 0 & $24 \pm 2^{\mathrm{A}}$ & $25 \pm 1^{\mathrm{A}}$ & $5 \pm 3^{\mathrm{A}}$ & $46 \pm 3^{A}$ & $25 \pm 1^{\mathrm{A}}$ & $21 \pm 3^{\mathrm{A}}$ \\
\hline 5 & $22 \pm 1^{\mathrm{A}}$ & $20 \pm 2^{\mathrm{A}}$ & $6 \pm 3^{\mathrm{A}}$ & $52 \pm 3^{\mathrm{A}}$ & $23 \pm 3^{\mathrm{A}}$ & $29 \pm 2^{\mathrm{AB}}$ \\
\hline 10 & $15 \pm 3^{\mathrm{B}}$ & $9 \pm 1^{\mathrm{B}}$ & $0 \pm 0^{\mathrm{A}}$ & $76 \pm 4^{\mathrm{B}}$ & $38 \pm 3^{\mathrm{B}}$ & $38 \pm 2^{\mathrm{B}}$ \\
\hline 20 & $23 \pm 0^{\mathrm{AB}}$ & $0 \pm 0^{\mathrm{C}}$ & $0 \pm 0^{\mathrm{A}}$ & $77 \pm 2^{\mathrm{B}}$ & $67 \pm 3^{C}$ & $10 \pm 1^{\mathrm{C}}$ \\
\hline 30 & $14 \pm 1^{\mathrm{B}}$ & $0 \pm 0^{\mathrm{C}}$ & $0 \pm 0^{\mathrm{A}}$ & $86 \pm 3^{B}$ & $86 \pm 3^{\mathrm{D}}$ & $0 \pm 0^{\mathrm{D}}$ \\
\hline
\end{tabular}

Oocytes were matured in vitro for $48 \mathrm{~h}$ and then further cultured for another 3 days with the respective concentrations of the JNK inhibitor. The total number of oocytes in each group is 120 . A,B,C,D Statistically significant differences between oocytes of the same type after different treatments with JNK inhibitor (i.e., differences within columns) are indicated by different superscripts $(\mathrm{P}<0.05)$. The same letters indicate statistical insignificance.

trations of inhibitors) were evaluated by analysis of variance (Scheffes' test) using the STATISTICA statistical software package (STATISTICA 8.0; StatSoft, Tulsa, OK, USA). A P value of less than 0.05 was considered significant.

Measurement of JNK and p38 MAPK activity was repeated four times. The differences between the groups of oocytes were evaluated by analysis of variance using the SAS statistical software package (SAS V9.1; SAS Institute, Cary, NC, USA). A P value of less than 0.05 was considered significant.

\section{Results}

Oocytes cultured in vitro for $48 \mathrm{~h}$ reached the stage of metaphase II in $98 \%$ of cases. The remaining oocytes were at the stage of metaphase I, anaphase I or telophase I.

Representative photographs of aged oocytes are shown in Fig. 1. During the first day of aging, the oocytes remained mostly at the stage of metaphase II. During the second day of aging, there was a significant decrease in oocytes remaining at the stage of metaphase II. Simultaneously, there was a significant increase in the ratio of oocytes that underwent spontaneous parthenogenetic activation or fragmentation. During the third day of oocyte aging, the ratio of oocytes at the stage of metaphase II further decreased, and the ratio of fragmented oocytes further increased. On the fourth day of aging, the ratio of parthenogenetically activated oocytes decreased as did the ratio of fragmented oocytes. There were no significant changes in oocytes on the fifth day of aging with the exception of lysed oocytes.

The most significant changes were observed on the third day of aging, when the ratio of metaphase II oocytes was low, but it was not significantly different from the MII ratio after 4 and 5 days of aging. The ratios of fragmented and spontaneously activated oocytes reached their maximums on the third day of aging. For these reasons, we used oocytes aged for 3 days in the subsequent experiments.

In Experiment 2, we investigated the effect of JNK inhibition on pig oocyte aging. The ratio of fragmented oocytes decreased in a dose-dependent manner after JNK inhibition using 1,9-pyrazoloanthrone (Table 2), and this indicated the involvement of JNK in oocyte fragmentation. At the same time, the ratio of the spontaneously parthenogenetically activated oocytes increased in a dosedependent manner. Up to $10 \mu \mathrm{M}$, the ratio of oocytes remaining at the stage of metaphase II and undergoing lysis did not change significantly. Oocytes with pronuclei or cleaved oocytes were designated as parthenogenetically activated. The ratio of oocytes to pronuclei significantly increased in a dose-dependent manner as the dose of JNK inhibitor increased. The ratio of cleaved oocytes increased with up to $10 \mu \mathrm{M}$ of the JNK inhibitor (38\% of all cleaved oocytes) and then decreased to $0 \%$ of cleaved oocytes at a concentration of $30 \mu \mathrm{M}$ of JNK inhibitor. Therefore, inhibition of JNK promoted oocyte activation but suppressed cleavage of parthenogenetic embryos.

Culture with an inactive analog of the JNK inhibitor $\mathrm{N}^{1}$-methyl1, 9-pyrazoloanthrone at concentrations of $0,5,10,20$ and $30 \mu \mathrm{M}$ 
Table 3. Effect of the p38 MAPK inhibitor 2-(4-chlorophenyl)-4-(4-fluorophenyl)-5-pyridin-4-yl-1,2 dihydropyrazol-3-one on the aging and spontaneous parthenogenetic activation of aged pig oocytes

\begin{tabular}{|c|c|c|c|c|c|c|}
\hline \multirow{2}{*}{$\begin{array}{l}\text { Concentration } \\
\text { of p38 MAPK } \\
\text { inhibitor }(\mu \mathrm{M})\end{array}$} & \multicolumn{5}{|c|}{ Type of oocyte (\% \pm SE) } & \multirow[b]{2}{*}{$\begin{array}{c}\text { Activated } \\
\text { (cleaved } \\
\text { oocytes) }\end{array}$} \\
\hline & Metaphase II & Fragmented & Lysed & $\begin{array}{c}\text { Activated } \\
\text { (sum) }\end{array}$ & $\begin{array}{c}\text { Activated } \\
\text { (oocytes with } \\
\text { pronuclei) }\end{array}$ & \\
\hline 0 & $20 \pm 3^{\mathrm{A}}$ & $29 \pm 1^{\mathrm{A}}$ & $7 \pm 2^{\mathrm{A}}$ & $44 \pm 4^{\mathrm{A}}$ & $22 \pm 2^{\mathrm{A}}$ & $22 \pm 2^{\mathrm{A}}$ \\
\hline 0.1 & $17 \pm 2^{\mathrm{A}}$ & $31 \pm 2^{\mathrm{A}}$ & $9 \pm 1^{\mathrm{A}}$ & $43 \pm 2^{\mathrm{A}}$ & $23 \pm 1^{\mathrm{A}}$ & $20 \pm 1^{\mathrm{AB}}$ \\
\hline 0.5 & $20 \pm 1^{\mathrm{A}}$ & $33 \pm 2^{A}$ & $6 \pm 3^{\mathrm{A}}$ & $41 \pm 2^{\mathrm{A}}$ & $30 \pm 2^{\mathrm{AB}}$ & $11 \pm 1^{\mathrm{C}}$ \\
\hline 1.0 & $18 \pm 1^{\mathrm{A}}$ & $32 \pm 1^{\mathrm{A}}$ & $2 \pm 1^{\mathrm{A}}$ & $48 \pm 1^{\mathrm{A}}$ & $35 \pm 2^{\mathrm{B}}$ & $13 \pm 1^{\mathrm{BC}}$ \\
\hline 2.5 & $15 \pm 1^{\mathrm{A}}$ & $34 \pm 2^{\mathrm{A}}$ & $7 \pm 2^{\mathrm{A}}$ & $44 \pm 3^{\mathrm{A}}$ & $35 \pm 3^{\mathrm{B}}$ & $9 \pm 2^{\mathrm{C}}$ \\
\hline 5 & $14 \pm 1.8^{\mathrm{A}}$ & $36 \pm 3.0^{\mathrm{A}}$ & $4 \pm 2^{\mathrm{A}}$ & $46 \pm 2^{\mathrm{A}}$ & $39 \pm 2^{\mathrm{B}}$ & $7 \pm 1^{\mathrm{C}}$ \\
\hline
\end{tabular}

Oocytes were matured in vitro for $48 \mathrm{~h}$ and then further cultured for another 3 days with the respective concentrations of the p38 MAPK inhibitor. The total number of oocytes in each group is 120 . ${ }^{\mathrm{A}, \mathrm{B}, \mathrm{C}}$ Statistically significant differences between oocytes of the same type after different treatments with the p38 MAPK inhibitor (i.e., within the columns) are indicated by different superscripts $(\mathrm{P}<0.05)$. The same letters indicate statistical insignificance.

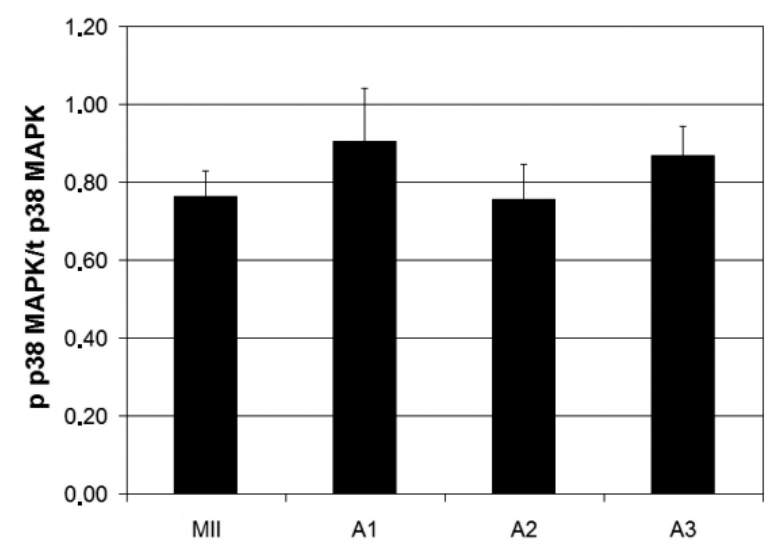

A

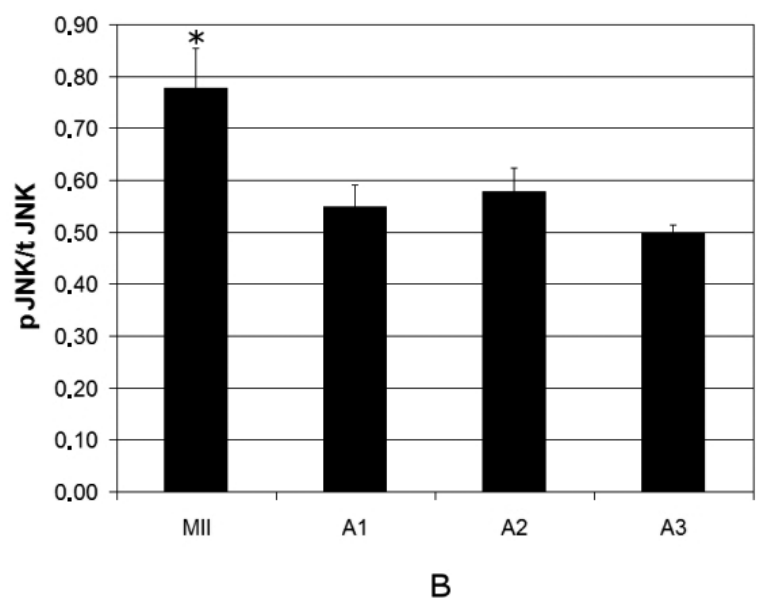

B

Fig. 2. Activity of p38 MAPK and JNK in aged pig oocytes. The ratio of phosphorylated p38 MAPK (p p38 MAPK) to total p38 MAPK ( $\mathrm{p}$ 38 MAPK) (A) and the ratio of phosphorylated JNK (p JNK) to total JNK ( $\mathrm{JNK}$ ) (B) are shown in the graphs. While the ratio p p38 MAPK/t p 38 MAPK was not significantly different in all groups of oocytes, the ratio p JNK/t JNK in all groups of aged oocytes (A1, A2 and A3) was significantly lower than that in MII oocytes $(*$ - significant difference, $\mathrm{P}<0.05)$. The values represent the means \pm SE. MII, A1, A2, A3-oocytes were aged for 0,1 , 2 or 3 days, respectively.

did not result in any change compared to oocyte aging in a medium without any supplementation. After 3 days of aging with the highest concentration of $\mathrm{N}^{1}$-methyl-1,9-pyrazoloanthrone (30 $\left.\mu \mathrm{M}\right)$, only $20 \%$ of the oocytes remained at the stage of metaphase II, $42 \%$ were spontaneously activated, $30 \%$ underwent fragmentation and 8\% were lysed.

Experiment 3 revealed no significant effect of inhibition of kinase p38 MAPK on the fragmentation of aged oocytes. The ratio of fragmented oocytes remained the same up to a concentration of $5 \mu \mathrm{M}$ of the p38 MAPK inhibitor 2-(4-chlorophenyl)-4-(4-fluorophenyl)-5-pyridin-4-yl-1,2 dihydropyrazol-3-one. At a higher concentration, the p38 MAPK inhibitor had detrimental effects because a significant portion of the oocytes underwent lysis (data not shown). Other categories of aged oocytes, i.e., activated, lysed or intact oocytes remaining at the stage of metaphase II, remained unchanged when exposed to the p38 MAPK inhibitor at concentra- tions of up to $5 \mu \mathrm{M}$ (Table 3). Similar to the inhibition of JNK, an increased dose of the p38 MAPK inhibitor significantly reduced the portion of cleaved parthenogenetic embryos and significantly increased the ratio of activated oocytes with pronuclei.

In Experiment 4, we determined the activity of JNK and p38 MAPK (Fig. 2). The ratio of the activated JNK to total JNK significantly decreased during the first day of aging compared with the MII oocyte ratio. This significant difference remained during the entire period of oocyte aging (Fig. 2B). The ratio of activated p38 MAPK to total p38 MAPK did not significantly change during aging of the pig oocytes (Fig. 2A).

In Experiment 5, the activity of JNK during activation of oocytes was determined. We compared MII oocytes with oocytes after $12 \mathrm{~h}$ of aging and with activated oocytes. No significant differences were found between the groups in terms of the ratio of activated JNK to total JNK (Fig. 3). 


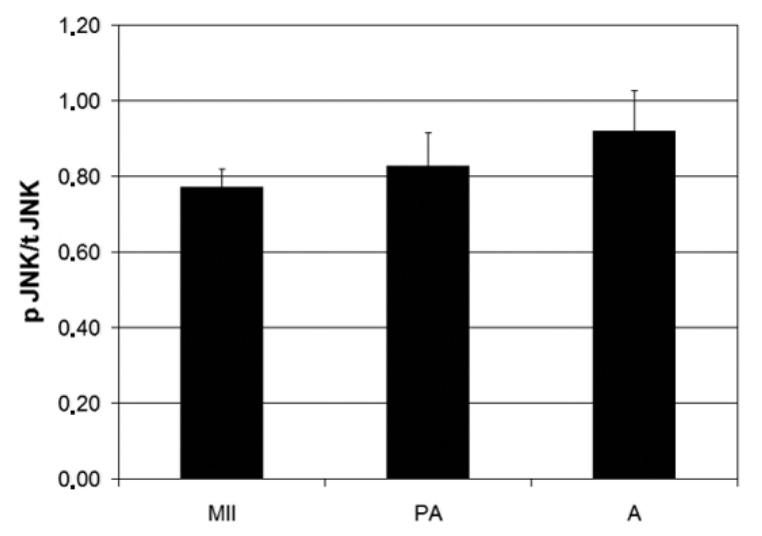

Fig. 3. The JNK activity of activated MII oocytes. The ratio of phosphorylated JNK (p JNK) to total JNK (t JNK) is shown in the graph. The ratio $\mathrm{p} \mathrm{JNK/t} \mathrm{JNK} \mathrm{was} \mathrm{not}$ significantly different in all groups of oocytes $(\mathrm{P}<0.05)$. The values represent means $\pm \mathrm{SE}$. MII-matured oocytes, PA-parthenogenetically activated oocytes (by calcium ionophore A23187 and 6-dimethylaminopurine) after 12 $\mathrm{h}$ of aging and A-oocytes aged for $12 \mathrm{~h}$.

In Experiment 6, subcellular localization of the phosphorylated form of JNK in aged oocytes was performed (Fig. 4). Phosphorylated JNK was localized in the cortex region in MII oocytes and under the plasma membrane in fragmented oocytes. Phosphorylated JNK was found to be localized under the plasma membrane of cleaved oocytes and was also concentrated in the nucleoli of embryos. However, only a weak signal was found in lysed oocytes.

\section{Discussion}

In the present study, we demonstrated the changes in porcine oocytes during aging under in vitro conditions and the effect of JNK and p38 MAPK inhibition on aging. JNK inhibition suppressed the fragmentation of in vitro aged pig oocytes, which is one of the manifestations of aging [16, 19, 20]. Inhibition of p38 MAPK did not induce a similar effect.

Oocyte aging is accompanied by inactivation of the cytostatic factor (CSF) [10], which stabilizes MPF and maintains the oocyte at the second meiotic arrest [33]. This CSF inactivation allows spontaneous parthenogenetic activation of aged oocytes. Inactivation of CSF and decrease of MPF could activate pro-apoptotic factors and/or could inactivate anti-apoptotic factors. When this occurs, aged oocytes become fragmented [34].

It seems that in contrast to starfish oocytes, in which apoptosis during aging depends on both JNK and p38 MAPK [17, 22], apoptosis of aged porcine oocytes is connected only with the activity of JNK. The reason for this difference is not clear. However, p38 MAPK can play a different role in aging in invertebrates and vertebrates, similar to that during meiosis. While entry to the first meiotic metaphase in the starfish oocyte depends on inactivation of the p38 MAPK homologue designated as Mipk [35], the p38 MAPK kinase is activated in the pig oocyte after resumption of meiosis, and it remains active during metaphase I up to metaphase
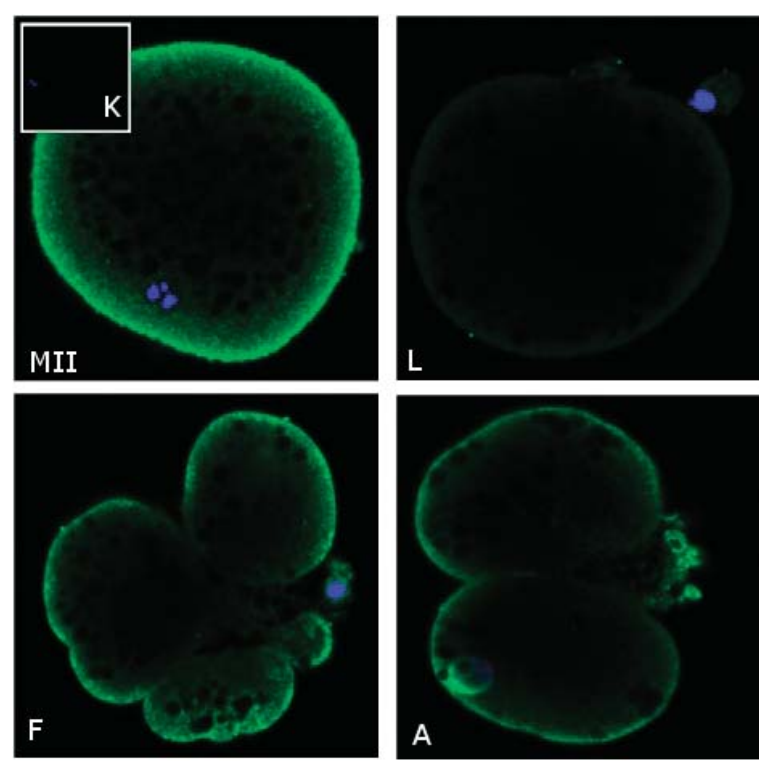

Fig. 4. Subcellular localization of phosphorylated JNK in aged pig oocytes. Representative pictures of the JNK localization are shown. MII-metaphase II oocyte (1 day of aging), K-MII (control oocyte exposed only to the second antibody), F-fragmented oocyte (3 days of aging), L-lysed oocyte (3 days of aging) and A-activated oocyte (cleaved oocyte, 3 days of aging). The oocytes were stained with mouse anti-phospho-JNK and anti mouse IgG-FITC (green) and Hoechst 33258 (blue). Magnification $40 \times$.

II [28].

The observed suppression of oocyte fragmentation after JNK inhibition is in accordance with the important role of JNK in apoptosis in both vertebrates and invertebrates. JNK is involved in signalling, which is induced by cell stress and triggers apoptosis [36]. However, the precise role of JNK in induction of apoptosis is not yet well understood. JNK may act through inhibition of the anti-apoptotic factors Bcl-2 and Bcl- $\mathrm{X}_{\mathrm{L}}$, which loose their anti-apoptotic effects after phosphorylation by JNK [36], or it may act through activation of the pro-apoptotic factors Bax, Bak, Bid or Bim [37-40].

JNK inhibition caused an increase in the portion of spontaneously activated oocytes in the present study, and this was accompanied by a significant reduction in cleaved parthenogenetic embryos. We can only speculate about the reasons for this effect. In mammalian oocytes, the arrest of meiosis at metaphase II is induced by activation of a complex formed by cyclin B and cyclindependent kinase Cdc2 and by stabilization of this complex (MPF) by CSF [41]. These events are dependent on the Mos-MAP kinase signalling pathway, which induces inhibition of the anaphase-promoting complex/cyclosome (APC/C) [41]. Exit from the stage of metaphase II is dependent on destabilization of the Cdc2-cyclin B complex and cyclin destruction [33]. JNK phosphorylates many different regulatory proteins, including the tumor suppressor protein p53 $[42,43]$. The protein p53 plays an important role in regulation of apoptosis [36] but is also known as an effective regu- 
lator of c-Mos protein [44], which is involved in establishment of meiotic blockage in matured oocytes at the stage of metaphase II. It is possible that p53 mediates the effect of JNK on CSF and spontaneous activation of aged oocytes. However, additional experiments are necessary for an explanation of the JNK-inhibitor effect on spontaneous parthenogenetic activation of aged oocytes and the embryos that originated from this activation.

Contrary to results for JNK, inhibition of p38 MAPK had no significant effect on the fragmentation of aging pig oocytes, nor did it have any significant effect on spontaneous activation of aging pig oocytes (Table 3). It only decreased parthenogenetic cleavage and increased the portion of oocytes that formed pronuclei. This indicates that inhibition of p38 MAPK did not prevent parthenogenesis, but instead suppressed cleavage of parthenogenetic embryos. This is no great surprise with respect to the well-known role of p38 MAPK in regulation of the cell cycle [45]. The final effect of p38 MAPK depends on the type of cell and type of stimulus. According to these conditions, inhibition of p38 MAPK induces blockage of the cell cycle at many different stages, such as entry, transition from the G1 to S phase and transition from the G2 to M phase [45]. Similar effects of p38 MAPK have been observed in embryos, but the data are contradictory. Natale et al. [46] observed blockage of development in mouse pre-implantation embryos after inhibition of p38 MAPK, while Wang et al. [47] observed the activity of p38 MAPK during in vitro culture of embryos. An increase of active forms of the kinase induces blockage of embryo development. On the other hand, Madan et al. [48] did not observe any effect of p38 MAPK inhibition on the pre-implantation development of cattle embryos.

Activated JNK and p38 MAPK were detected in pig oocytes matured in vitro and in aging pig oocytes. The phosphorylated form of JNK was localized in parthenogenetic embryos and fragmented oocytes under the plasma membrane and nucleus. We did not detect it in lysed oocytes. While the ratio of activated p38 MAPK to total p38 MAPK did not change during aging, the ratio of activated JNK to total JNK decreased. This decrease was probably not due to the increased portion of spontaneously activated oocytes because parthenogenetic activation of oocytes by calcium ionophore was not associated with the change in JNK activity, and phosphorylated JNK was present in the parthenogenetic embryos. A decrease in the ratio of activated JNK to total JNK could be caused by loss of JNK activity in lysed oocytes, which was observed in our experiment during aging. Based on these data, we can assume that the decrease in JNK activity was caused by the aging of oocytes only and not by their exit from the metaphase II stage.

JNK activity does not change after oocyte activation, but significantly decreases during further oocyte aging. We can speculate that even this decreased activity of JNK is sufficient to trigger apoptosis. Further decrease of JNK activity using a specific JNK inhibitor could prevent the onset of apoptosis. The activity of p38 MAPK has no significant effect on the fragmentation of aging pig oocytes.

Based on our experiments, it can be concluded that fragmentation of aging pig oocytes is induced by active JNK. Morphological cell changes like membrane blebbing and apoptotic body formation are JNK-dependent processes [49], and we detected the phosphorylated form of JNK in fragmented oocytes. However, further experiments are necessary for exact elucidation of the JNK role in this process because JNK isoforms can play different roles in these cells. While one isoform can be translocated to the nucleus, the others remain localized in the cytoplasm [50] or can be associated with mitochondrial membranes [51]. The primary antibody used in our experiment reacts with activated diphosphorylated (Thr ${ }^{138}$ and Tyr ${ }^{185}$ ) forms of all isoforms of JNK. Although the antibody does not recognize any form of p38 MAPK, it can weakly cross-react with non-phosphorylated or monophosphorylated tyrosin peptides of JNK.

Further studies are needed for elucidation of the processes involved in the aging of mammalian oocytes. However, inhibition of JNK seems to be a possible way of preventing detrimental processes accompanying the aging of mammalian oocytes.

\section{Acknowledgments}

We thank Mrs. L Westcott and Mrs. L Russell for their editorial assistance with this manuscript. This project was supported by grants NAZV QG 50052, MZE 0002701401 , MSM 6046070901 and GACR 523/08/H064.

\section{References}

1. Wassarman PM. The mammalian ovum. In: Knobil E, Neil J (eds.), The Physiology of Reproduction. New York: Raven Press; 1988: 69-102.

2. Yanagimachi R. Mammalian fertilization. In: Knobil E, Neil J (eds), The Physiology of Reproduction. New York: Raven Press; 1988: 135-185.

3. Chian RC, Nakahara H, Niwa K, Funahashi H. Fertilization and early cleavage in vitro of aging bovine oocytes after maturation in culture. Theriogenology 1992; 37: 665672.

4. Pavlok A, Kalab P, Bobak P. Fertilisation competence of bovine normally matured or aged oocytes derived from different antral follicles: Morphology, protein synthesis, H1 and MBP kinase activity. Zygote 1997; 5: 235-246.

5. Stice SL, Keefer CL, Matthews L. Bovine nuclear transfer embryos: oocyte activation prior to blastomere fusion. Mol Reprod Dev 1994; 38: 61-68.

6. Tanaka H, Kanagawa $\mathbf{H}$. Influence of combined activation treatments on the success of bovine nuclear transfer using young or aged oocytes. Anim Reprod Sci 1997; 49: 113123

7. Iwamoto M, Onishi A, Fuchimoto D, Somfai T, Suzuki S, Yazaki S, Hashimoto M, Takeda K, Tagami T, Hanada H, Noguchi J, Kaneko H, Nagai T, Kikuchi K. Effects of caffeine treatment on aged porcine oocytes: parthenogenetic activation ability, chromosome condensation, and development to the blastocyst stage after somatic cell nuclear transfer. Zygote 2005; 13: 335-345.

8. Jolliff WJ, Prather RS. Parthenogenic development of in vitro-matured, in vivo-cultured porcine oocytes beyond blastocyst. Biol Reprod 1997; 56: 544-548.

9. Suzuki H, Takashima Y, Toyokawa K. Cytoskeletal organization of porcine oocytes aged and activated electrically or by sperm. J Reprod Dev 2002; 48: 293-301.

10. Kikuchi K, Naito K, Noguchi J, Shimada A, Kaneko H, Yamashita M, Aoki F, Tojo H, Toyoda Y. Maturation/M-phase promoting factor: A regulator of aging in porcine oocytes. Biol Reprod 2000; 63: 715-722.

11. Petrova I, Sedmikova M, Chmelikova E, Svestkova D, Rajmon R. In vitro aging of porcine oocytes. Czech J Anim Sci 2004; 49: 93-98.

12. Petrova I, Rajmon R, Sedmikova M, Kuthanova Z, Jilek F, Rozinek J. Improvement of developmental competence of aged porcine oocytes by means of the synergistic effect of insulin-like growth factor-1 (IGF-1) and epidermal growth factor (EGF). Czech J Anim Sci 2005; 50: 300-310.

13. Lim E-A, Choi T-S. A phenotypic study of murine oocyte death in vivo. J Reprod Dev 2004; 50: 179-183.

14. Liu G, Kato Y, Tsunoda Y. Aging of recipient oocytes reduces the development of cloned embryos receiving cumulus cells. J Reprod Dev 2007; 53: 785-790.

15. Austin CR. Ageing and reproduction: post-ovulatory deterioration of the egg. J Reprod Fertil (Suppl) 1970; 12: 39-53. 
16. Fujino Y, Ozaki K, Yamamasu S, Ito F, Matsuoka I, Hayashi E, Nakamura H, Ogita S, Sato E, Inoue M. DNA fragmentation of oocytes in aged mice. Hum Reprod 1996; 11: 1480-1483.

17. Sasaki K, Chiba K. Fertilization blocks apoptosis of starfish eggs by inactivation of the MAP kinase pathway. Dev Biol 2001; 237: 18-28.

18. Yüce $\mathrm{O}$, Sadler KC. Postmeiotic unfertilized starfish eggs die by apoptosis. Dev Biol 2001; 237: 29-44.

19. Takase K, Ishikawa M, Hoshiai H. Apoptosis in the degeneration process of unfertilized mouse ova. Tohoku J Exp Med 1995; 175: 69-76.

20. Tarin JJ, Perez-Albala S, Cano A. Cellular and morphological traits of oocytes retrieved from aging mice after exogenous ovarian stimulation. Biol Reprod 2001; 65: 141-150.

21. Adams JM, Cory S. The Bcl-2 protein family: Arbiters of cell survival. Science 1998; 281: 1322-1326.

22. Sadler KC, Yuce O, Hamaratoglu F, Verge V, Peaucellier G, Picard A. MAP kinases regulate unfertilized egg apoptosis and fertilization suppresses death via $\mathrm{Ca}^{2+}$ signaling. Mol Reprod Dev 2004; 67: 366-383.

23. Kyriakis JM, Avruch J. Mammalian mitogen-activated protein kinase signal transduction pathways activated by stress and inflammation. Physiol Rev 2001; 81: 807-869.

24. Roux PP, Blenis J. ERK and p38 MAPK-activated protein kinases: A family of protein kinases with diverse biological functions. Microbiol Mol Biol Rev 2004; 68: 320-344.

25. Pearson G, Robinson F, Beers Gibson T, Xu BE, Karandikar M, Berman K, Cobb MH. Mitogen-activated protein (MAP) kinase pathways: Regulation and physiological functions. Endocr Rev 2001; 22: 153-183.

26. Mood K, Bong YS, Lee HS, Ishimura A, Daar IO. Contribution of JNK, Mek, Mos and PI-3K signaling to GVBD in Xenopus oocytes. Cell Signal 2004; 16: 631-642.

27. Baatout S, Jaussi R, Michaux A, Buset J, Schoonjans W, Jacquet P. Intracellular signal transduction in mouse oocytes and irradiated early embryos. In Vivo 2007; 21: 587592.

28. Villa-Diaz LG, Miyano T. Activation of p38 MAPK during porcine oocyte maturation. Biol Reprod 2004; 71: 691-696.

29. Xia Z, Dickens M, Raingeaud J, Davis RJ, Greenberg ME. Opposing effects of ERK and JNK-p38 MAP kinases on apoptosis. Science 1995; 270: 1326-1331.

30. Zhang Y, Huang Y, Rishi AK, Sheikh MS, Shroot B, Reichert U, Dawson M, Poirer G, Fontana JA. Activation of the p38 and JNK/SAPK mitogen-activated protein kinase pathways during apoptosis is mediated by a novel retinoid. Exp Cell Res 1999; 247: 233-240.

31. Assefa Z, Vantieghem A, Garmyn M, Declercq W, Vandenabeele P, Vandenheede JR, Bouillon R, Merlevede W, Agostinis P. p38 mitogen-activated protein kinase regulates a novel, caspase-independent pathway for the mitochondrial cytochrome $\mathrm{C}$ release in ultraviolet B radiation-induced apoptosis. J Biol Chem 2000; 275: 2141621421.

32. Jilek F, Hüttelova R, Petr J, Holubova M, Rozinek J. Activation of pig oocytes using calcium ionophore: effect of the protein kinase inhibitor 6-dimethyl aminopurine. Reprod Domest Anim 2001; 36: 39-145.

33. Maller JL, Schwab MS, Gross SD, Taieb FE, Roberts BT, Tunquist BJ. The mechanism of CSF arrest in vertebrate oocytes. Mol Cell Endocrinol 2002; 187: 173-178.

34. Fissore A, Kurokawa M, Knott J, Zhang M, Smyth J. Mechanisms underlying oocyte activation and postovulatory ageing. Reproduction 2002; 124, 745-754

35. Morrison DL, Yee A, Paddon HB, Vilimek D, Aebersold R, Pelech SL. Regulation of the meiosis-inhibited protein kinase, a p38(MAPK) isoform, during meiosis and following fertilization of seastar oocytes. J Biol Chem 2000; 275: 34236-34244.

36. Kanda H, Miura M. Regulatory roles of JNK in programmed cell death. J Biochem 2004; 136: 1-6.

37. Lei K, Nimnual A, Zong WX, Kennedy NJ, Flavell RA, Thompson CB, Bar-Sagi D, Davis RJ. The Bax subfamily of Bcl2-related proteins is essential for apoptotic signal transduction by c-Jun NH(2)-terminal kinase. Mol Cell Biol 2002; 22: 4929-4942.

38. Lei K, Davis RJ. JNK phosphorylation of Bim-related members of the Bcl2 family induces Bax-dependent apoptosis. Proc Natl Acad Sci USA 2003; 100: 2432-2437.

39. Putcha GV, Le S, Frank S, Besirli CG, Clark K, Chu B, Alix S, Youle RJ, LaMarche A, Maroney AC, Johnson EM Jr. JNK-mediated BIM phosphorylation potentiates BAXdependent apoptosis. Neuron 2003; 38: 899-914.

40. Papadakis ES, Finegan KG, Wang X, Robinson AC, Guo C, Kayahara M, Tournier C. The regulation of Bax by c-Jun $\mathrm{N}$-terminal protein kinase (JNK) is a prerequisite to the mitochondrial-induced apoptotic pathway. FEBS Lett 2006; 580: 1320-1326.

41. Kishimoto T. Cell-cycle control during meiotic maturation. Curr Opin Cell Biol 2003, 15: 654-663.

42. Nishina H, Wada T, Katada T. Physiological roles of SAPK/JNK signaling pathway. Biochem 2004; 136: 123-126.

43. Vlahopoulos S, Zoumpourlis VC. JNK: a key modulator of intracellular signaling. Biochemistry 2004; 69: 844-854.

44. Gorgoulis VG, Zacharatos P, Mariatos G, Liloglou T, Kokotas S, Kastrinakis N, Kotsinas A, Athanasiou A, Foukas P, Zoumpourlis V, Kletsas D, Ikonomopoulos J, Asimacopoulos PJ, Kittas C, Field JK. Deregulated expression of c-mos in non-small cell lung carcinomas: Relationship with p53 status, genomic instability, and tumor kinetics. Cancer Res 2001; 61: 538-549.

45. Ambrosino C, Nebreda AR. Cell cycle regulation by p38 MAP kinases. Biol Cell 2001; 93: 47-51.

46. Natale DR, Paliga AJ, Beier F, D'Souza SJ, Watson AJ. p38 MAPK signaling during murine preimplantation development. Dev Biol 2004; 268: 76-88.

47. Wang Y, Puscheck EE, Lewis JJ, Trostinskaia AB, Wang F, Rappolee DA. Increase in phosphorylation of SAPK/JNK and p38 MAPK correlate negatively with mouse embryo development after culture in different media. Fertil Steril 2005; 83: 1144-1154.

48. Madan P, Calder MD, Watson AJ. Mitogen-activated protein kinase (MAPK) blockade of bovine preimplantation embryogenesis requires inhibition of both $\mathrm{p} 38$ and extracellular signal-regulated kinase (ERK) pathways. Reproduction 2005; 130: 41-51.

49. Zihni C, Mitsopoulos C, Tavares IA, Baum B, Ridley AJ, Morris JDH. Prostatederived sterile 20-like kinase 1-alpha induces apoptosis - JNK- and caspase-dependent nuclear localization is a requirement for membrane blebbing. J Biol Chem 2007; 282 6484-6493.

50. Katagiri C, Negishi K, Hibino T. c-JUN N-terminal kinase-1 (JNK1) but not JNK2 or JNK3 is involved in UV signal transduction in human epidermis. J Dermatol Sci 2006; 43: 171-179.

51. Zhou Q, Lam PY, Han D, Cadenas E. c-Jun N-terminal kinase regulates mitochondrial bioenergetics by modulating pyruvate dehydrogenase activity in primary cortical neurons. J Neurochem 2008; 104: 325-335. 